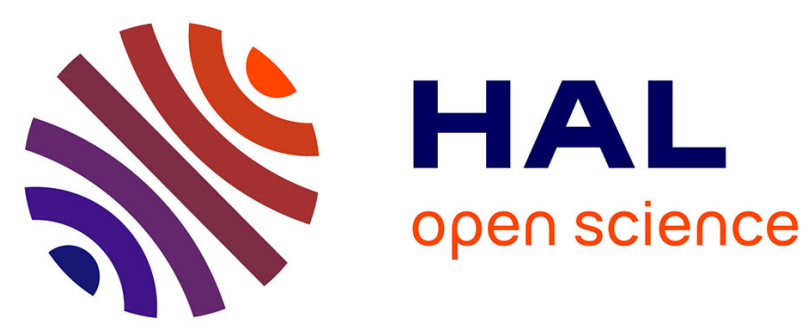

\title{
A first full wave simulation assessment of reflectometry for DTT
}

Filipe da Silva, Jorge Ferreira, G de Masi, Stéphane Heuraux, Emanuel Ricardo, T. Ribeiro, O Tudisco, R Cavazzana, O d'Arcangelo, Antonio Silva

\section{To cite this version:}

Filipe da Silva, Jorge Ferreira, G de Masi, Stéphane Heuraux, Emanuel Ricardo, et al.. A first full wave simulation assessment of reflectometry for DTT. Journal of Instrumentation, 2019, 14 (08), pp.C08011. 10.1088/1748-0221/14/08/C08011 . hal-02440059

\section{HAL Id: hal-02440059 \\ https://hal.univ-lorraine.fr/hal-02440059}

Submitted on 14 Jan 2020

HAL is a multi-disciplinary open access archive for the deposit and dissemination of scientific research documents, whether they are published or not. The documents may come from teaching and research institutions in France or abroad, or from public or private research centers.
L'archive ouverte pluridisciplinaire HAL, est destinée au dépôt et à la diffusion de documents scientifiques de niveau recherche, publiés ou non, émanant des établissements d'enseignement et de recherche français ou étrangers, des laboratoires publics ou privés. 


\title{
A first full wave simulation assessment of reflectometry for DTT
}

\author{
F. da Silva ${ }^{a}$ J. Ferreira, ${ }^{a}$, G. De Masi, ${ }^{b}$ S. Heuraux, ${ }^{c}$ E. Ricardo, ${ }^{a}$ T. Ribeiro, ${ }^{d}$ O. Tudisco, ${ }^{e}$ \\ R.Cavazzana, ${ }^{b}$ O. D'Arcangelo ${ }^{e}$ and A. Silva ${ }^{a}$ \\ ${ }^{a}$ Instituto de Plasmas e Fusão Nuclear, Instituto Superior Técnico, Universidade de Lisboa, \\ 1049-001 Lisboa, Portugal \\ ${ }^{b}$ Consorzio $R F X$, \\ Corso Stati Uniti, 4, 35127 Padova, Italy \\ ${ }^{c}$ Institut Jean Lamour UMR 7198 CNRS-Université de Lorraine, \\ ARTEM BP 50840, F-54011 Nancy, France \\ ${ }^{d}$ Max-Planck-Institut für Plasmaphysik, \\ Garching D-85748, Germany \\ ${ }^{e}$ Associazione EURATOM-ENEA-CR, \\ 00044 Frascati, Italy \\ E-mail: jferreira@ipfn.tecnico.ulisboa.pt
}

AbSTRACT: The Divertor Test Tokamak (DTT) facility is being proposed to study the exhaust solutions in tokamaks with a special look on DEMO, searching for alternatives to ITER's detached condition operation, for the case this solution proves inadequate. To know about the physical processes occurring in the plasma or for engineering needs and control uses, diagnostics play a major role and reflectometry, foreseen in the forthcoming generation of machines such as ITER and DEMO, is a useful asset for DTT. Assessing the different areas and applications that could be deployed on DTT is of significant importance from an early stage. We propose, therefore, to use numerical simulation using finite-difference time-domain codes (FDTD) of the REFMUL* family to implement synthetic reflectometry diagnostics. Different aspects are included such as propagation in the plasma, optimisation of the system location within the vacuum vessel, its access to the plasma (waveguide and antennas), or the signal processing techniques.

KeYwords: Simulation methods and programs; Plasma diagnostics - interferometry, spectroscopy and imaging

This is an author-created, un-copyedited version of an article accepted for publication/published in Journal of Instrumentation. IOP Publishing Ltd is not responsible for any errors or omissions in this version of the manuscript or any version derived from it. The Version of Record is available online at 10.1088/1748-0221/14/08/C08011. 


\section{Contents}

1 Introduction 1

2 Microwave reflectometry 1

$3 \quad$ Inputs to setup of a synthetic reflectometer using REFMUL 2

4 DDT synthetic reflectometers setup and simulation results 3

5 Discussion 4

\section{Introduction}

The Divertor Test Tokamak (DTT) facility is a new project for a machine intended to study the exhaust solutions in tokamaks with a special look on DEMO. ITER will operate in a detached condition, having a radiating volume in front of the divertor [1]. However, this solution may prove inadequate, and consequently, the need for sound alternatives is felt. As in all fusion machines, there will be a need for diagnostics to gather knowledge about the physical processes occurring in the plasma, for engineering needs and control. Reflectometry has become one of the most important techniques to diagnose fusion plasmas, and consequently, its use is foreseen in the forthcoming generation of machines such as ITER and DEMO. Also, for DTT, this diagnostic can be a useful asset. Therefore, assessing the different applications that could be deployed on DTT is of major importance. We propose to use numerical simulation using finite-difference time-domain codes (FDTD) of the REFMUL* family to implement synthetic reflectometry diagnostics which include aspects such as propagation in the plasma, optimisation of the system location within the vacuum vessel, its access to the plasma (waveguide and antennas), or the signal processing techniques. This study on the application of reflectometry to DTT happens in an early stage of the diagnostics' conception, and it is planned to be an aid to this process and a way to foment discussion. There are several pieces of information still not available at this moment, making this effort the start point of an evolving work. The applications developed to put the synthetic reflectometers in place will continue to be used as more details are made available.

\section{Microwave reflectometry}

Reflectometry is a spinoff of the radar techniques used in the study of the ionosphere, which has found an important application as a diagnostic in fusion research [2]. A signal is sent through the plasma where it propagates until being reflected at cutoff position $r_{c}$, where the refraction index is zero, $N\left(x_{c}\right)=0$. The reflected wave shows a phase shift $\varphi$ due to: (i) the microwave circuit $\varphi_{\mu}$; (ii) the propagation in a vacuum between the antenna and the plasma edge $\varphi_{0}$ and (iii) to 
the propagation in the plasma $\varphi_{p}$. The phase $\varphi_{p}$ reflects the propagation of the wave along a path, described in a cold plasma approximation by a refraction index $N(r)$ only, and it contains information on the plasma electronic density $n_{e}$. For the Ordinary mode (O-mode), $N$ is a function of the density $\varphi_{p}=\varphi_{p}\left[f, N_{O}\left(n_{e}\right)\right]$ while for the Extraordinary mode (X-mode) is also a function of the magnetic field $\varphi_{p}=\varphi_{p}\left[f, N_{X}\left(n_{e}, B_{0}\right)\right]$. The main computational electromagnetics (CEM) technique used to simulate reflectometry is finite-difference time-domain (FDTD), most commonly applied to Maxwell's curl equations, although it can also be applied to the wave equation [3-5]. It is usual to write the curl equation in a vacuum (with $\varepsilon_{0}$ and $\mu_{0}$ ) while including the plasma response in the current density $\mathbf{J}$. This results in a system coupling Maxwell equations with a linear differential equation (LDE) for $\mathbf{J}=\sigma \mathbf{E}$. To solve it, curl equations are discretised following the Yee schema while a time integrator is used to solve the LDE [6]. The REFMUL* family of FDTD codes comprises REFMUL (2D Ordinary mode-O-mode) [7], REFMULX/REFMULXp (2D Extraordinary mode) [8], REFMULF (2D full polarisation) and REFMUL3 (3D code) [9]. In this work we are using REFMUL, which has been used extensively in the simulation of ITER plasma position reflectometers [10].

\section{Inputs to setup of a synthetic reflectometer using REFMUL}

For preparing the synthetic reflectometers, some a priori information and data must be made available. A CAD description of the vessel is necessary to design the reflectometer accesses to the plasma and the shape of the first wall in the region of interest of the reflectometer together with the planned antenna design. Another point is to know how recessed the antennas need to be due to thermal and erosion protection. With this information in mind, the physical structure of the synthetic reflectometer can be implemented on REFMUL with the surfaces modelled as perfect electric conductors. For plasma modelling, knowledge of the equilibrium for the contemplated scenario has to be available comprising a $2 \mathrm{D}$ flux description $\Psi(R, Z)$ in machine coordinates that together with the evolution of the electron density profile on a magnetic coordinate, e.g. $n_{e}\left(\rho_{\text {pol }}\right)$, can be used to obtain the $2 \mathrm{D}$ density description $n_{e}(R, Z)$. At the time of this work, a description of $n_{e}$ fully consistent with the given EQDSK equilibrium is not yet available and therefore, an educated guess model had to be implemented. It was based on the density profiles appearing in [11] using a fit on the extracted data with the expression proposed in [12]

$$
\operatorname{mtanh}^{\mathrm{SOL}}(r)=\frac{h}{2}\left[\frac{\left(1+s^{\mathrm{CORE}} x\right) \mathrm{e}^{x}-\left(1+s^{\mathrm{SOL}}\right) \mathrm{e}^{-x}}{\mathrm{e}^{x}+\mathrm{e}^{-x}}+1\right] \text {, with } x=\frac{p_{\mathrm{pos}}-r}{2 \omega_{r}},
$$

where $r$ is the radius, $h$ is the pedestal height, $\omega_{r}$ is the pedestal width, and $p_{\text {pos }}$ is the pedestal position, all in real space along a line-of-sight.

In figure 1 we present a CAD model of DTT with the magnetic flux $\Psi(R, Z)$ superimposed on the left and on the right the adopted density model. This curve can now be used to map the $2 \mathrm{D}$ flux description $\Psi(R, Z)$ into a $2 \mathrm{D}$ density $n_{e}(R, Z)$. For each synthetic reflectometer, we do not need the full poloidal section of the torus, only a region of interest (ROI) enough to contain and describe the wave fields that describe the behaviour of the reflectometer. The ROI needs to be interpolated up to the resolution (number of points/wave length) used by REFMUL, in the case of this simulation, 20 points/wave length. 

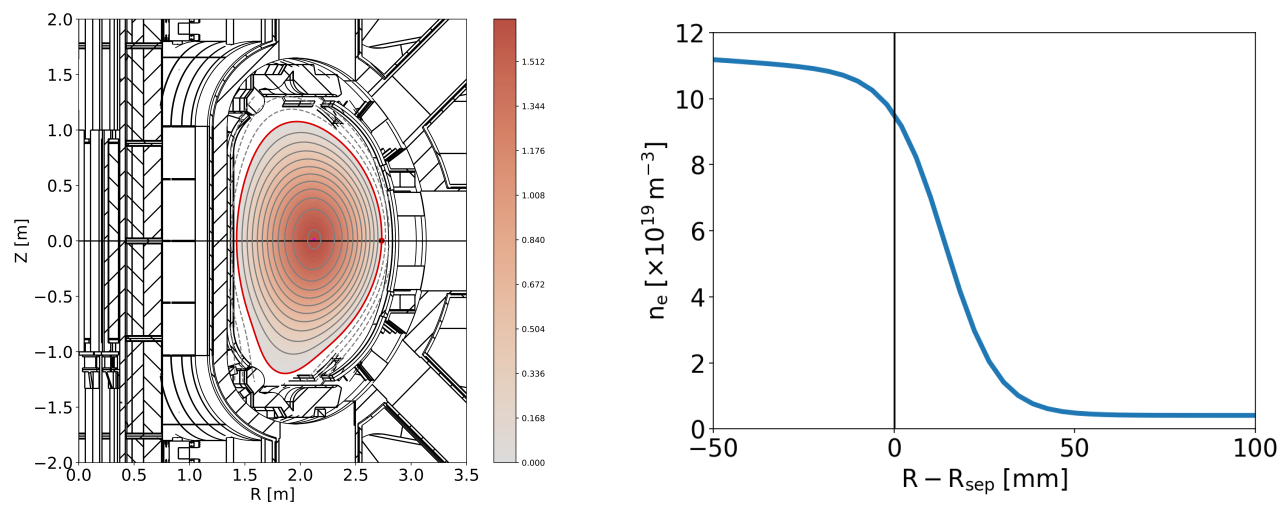

Figure 1. The input information needed to setup synthetic diagnostics comprises a description of the machine and an equilibrium scenario model. In the left we display a current design of DTT with a 2D map of the poloidal flux. The red line marks the separatrix. To obtain a 2D description of the density a model of $n_{e}$ along a magnetic coordinate is also needed. The density model used for this work appears on the right represented along the radial machine coordinate $R$. $R_{\text {sep }}$ is the position of the separatrix.

\section{DDT synthetic reflectometers setup and simulation results}

Two reflectometers were simulated, both on the low field side (LFS) of the vessel. One standard reflectometer placed at an equatorial position at an angle of $\alpha=0^{\circ}$ with a horizontal Line of Sight (LoS), and another at a lower position below the equatorial plane at an angle of $\alpha=-70^{\circ}$ with the horizontal LoS, being perpendicular to the separatrix. The antenna used was a $2 \mathrm{D}$ pyramidal horn antenna with a flare angle of $\psi_{h}=9^{\circ}$ (angle between the horn flare and the axis) and a length of $p=100$ grid points retracted into the vessel wall. The plasma is probed using frequency modulated continuous wave (FMCW) in the Ka band from $30 \mathrm{GHz}$ to $40 \mathrm{GHz}$ which covers a density range of $1.1-2 \times 10^{19} \mathrm{~m}^{-3}$. In figure 2 the snapshots of the synthetic reflectometers are displayed, on the left $\alpha=0^{\circ}$ and on the right $\alpha=-70^{\circ}$, they are presented not on the machine's $(R, Z)$ coordinate system but on REFMUL's $(x, y)$ were, for each case, the $x$-direction is made to coincide with the LoS of the antenna. We can observe the antenna access to the plasma through an aperture in the vessel. From each simulation the reflected signal is picked up in the waveguide, decoupled from the emitted signal though the use of an Unidirectional Transparent Source [7]. Through an
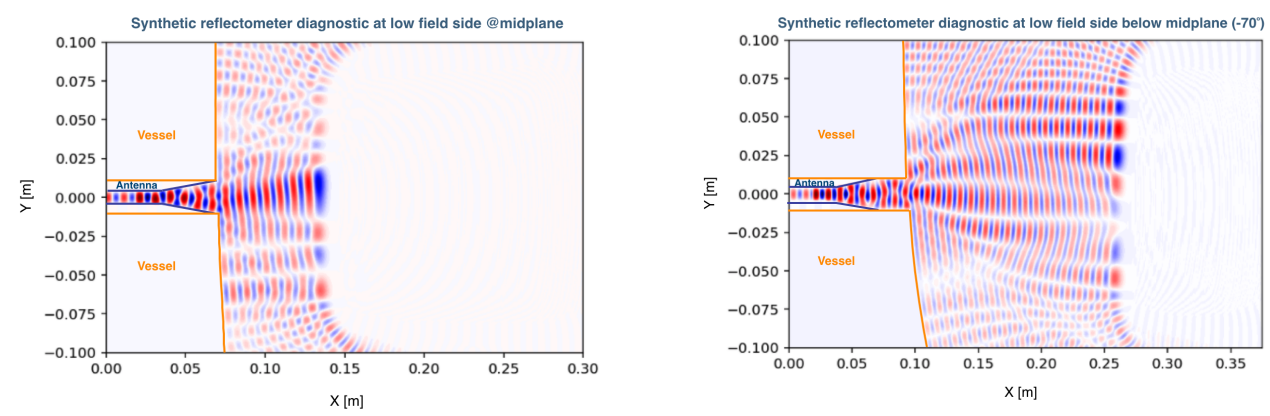

Figure 2. Synthetic reflectometers for DTT at the LFS, on the left a diagnostic at the midplane while on the right the diagnostic is setup below the middle plane looking perpendicular to the separatrix. 
In-phase/Quadrature detection (I/Q), the pair of reflectometer signals obtained can be used to get the phase signal $\varphi$ and from it the phase derivative $\partial \varphi / \partial f$. Alternatively a Sliding Fast Fourier Transform (SFFT) technique can be applied to one of the signals to obtain the beat frequency $f_{B}$ and from it $\partial \varphi / \partial f$. For the reflectometer in the equatorial plane, $\alpha=0^{\circ}$, figure 3 shows the results for the LFS midplane reflectometer: (i) on the top-left, the electric field $E_{z}$ detected in the waveguide; (ii) on the top-right, the pair of I/Q reflectometry signals. The shadow beneath shows the temporal (iterations) correspondence between the I/Q reflectometry signals and the $E_{z}$ field detected at the antenna; (iii) on the bottom-left the Sliding Fast Fourier Transform (SFFT) map of a I/Q IF reflectometry signal with the $f_{B}$ marked by the red dotted line and; (iv) the $\partial \varphi / \partial f$ obtained from I/Q detection and from SFFT technique. The equivalent results for the $\alpha=-70^{\circ}$ appear in figure 4 .
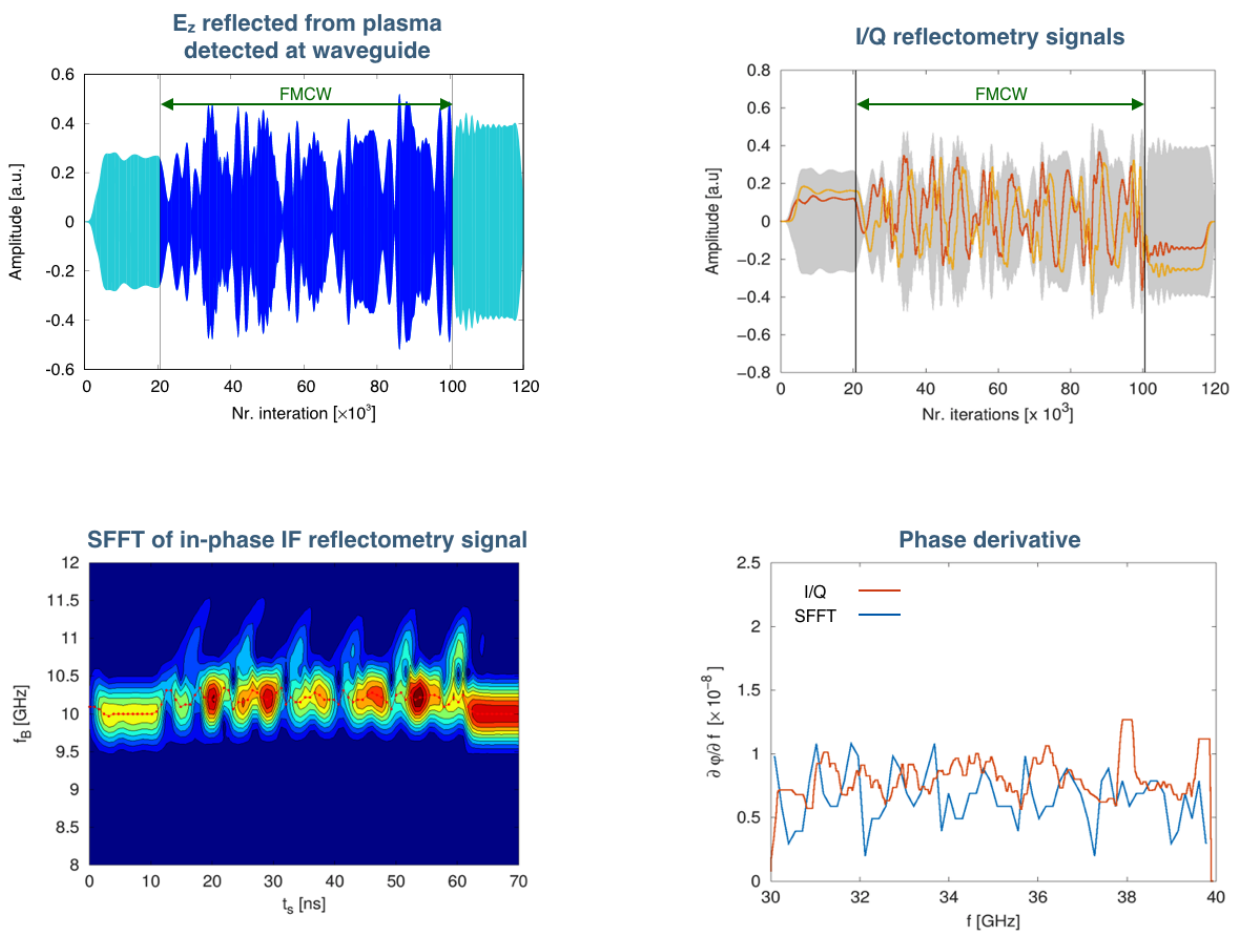

Figure 3. Signal processing of the results from the LFS midplane reflectometer $\left(\alpha=0^{\circ}\right)$. Top-left the electric field $E_{z}$ detected in the waveguide; top-right, the pair of I/Q reflectometry signals; bottom-left, the SFFT map of a I/Q IF reflectometry signal and; (iv) the $\partial \varphi / \partial f$ obtained from I/Q detection and from SFFT technique.

\section{Discussion}

Reflectometry will play a crucial role as a diagnostic for plasma position and pedestal density reconstruction for next-generation long-pulse fusion reactors, making it is essential to evaluate their viability rigorously during the design phase. Within this philosophy, we performed a first assessment of the viability of a reflectometry system for DTT and put together the utilities to adopt the input data (CAD and plasma description) to the simulation codes and prepare synthetic reflectometers 

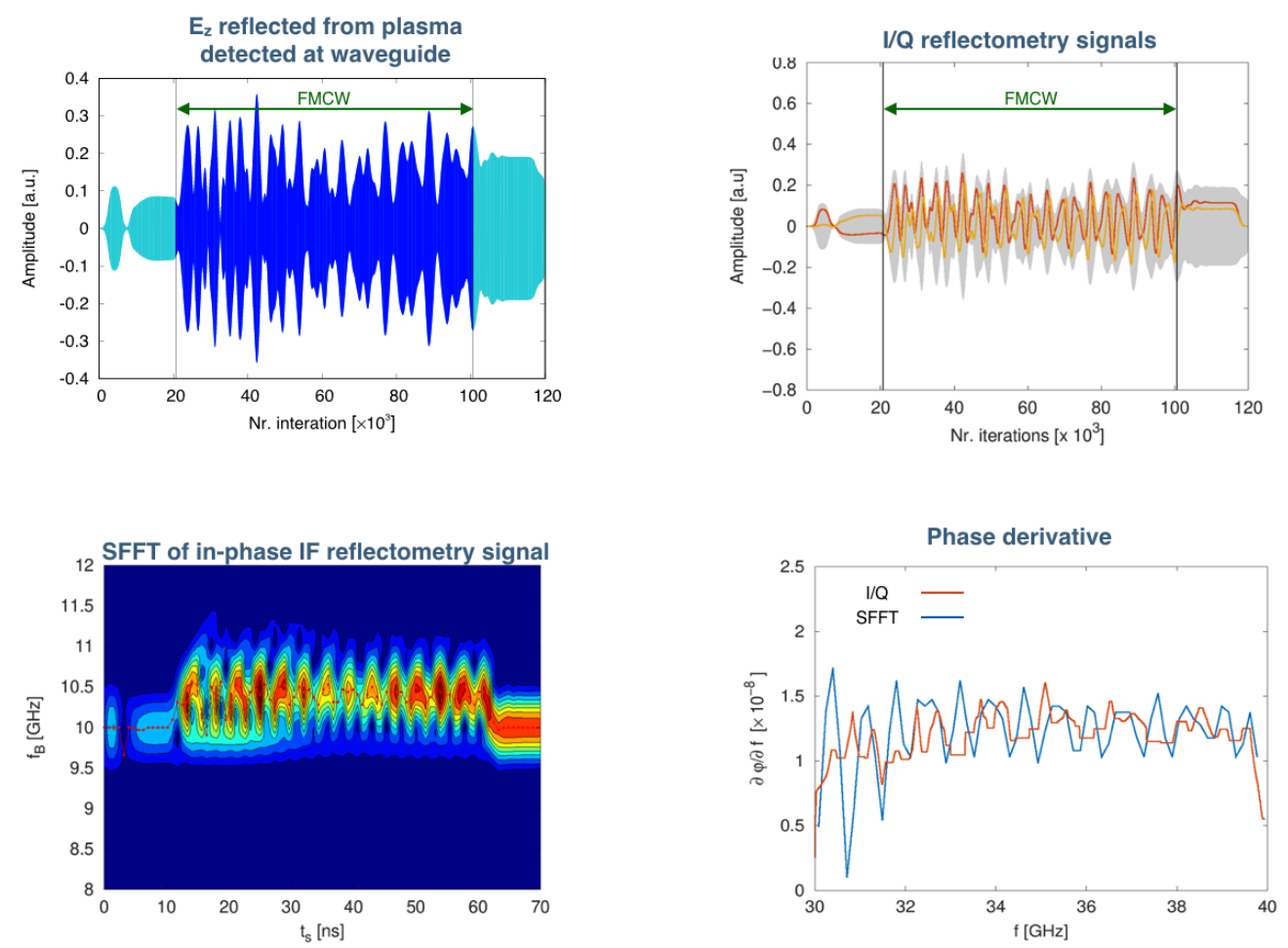

Figure 4. Signal processing of the results from the LFS below midplane reflectometer $\left(\alpha=-70^{\circ}\right)$. Top-left the electric field $E_{z}$ detected in the waveguide; top-right, the pair of I/Q reflectometry signals; bottom-left, the SFFT map of a I/Q IF reflectometry signal and; (iv) the $\partial \varphi / \partial f$ obtained from I/Q detection and from SFFT technique.

for DTT. This work also serves to help to identify the quality of the available information and its shortcomings. This is important to give feedback to DTT developers and to guarantee the continuity and evolution of this effort. Two reflectometry systems are planed for DTT, at present, for density profile reconstruction and turbulence studies although the details of the two systems are not fully decided. In the low magnetic field scenario, extraordinary X-mode will be probably used.

With this work, we explore the possibility of extending this scope since reflectometry in DTT could have a broader impact than on DTT itself. Implementation of reflectometry on DEMO for non-magnetic equilibrium control must be preceded by a phase of technical demonstration and validation able to establish it as a viable solution. That can only be fully accomplished with a previous installation and testing of reflectometry systems on existing or soon to be available machines, such as DTT. That would allow to test if reflectometry can be fully equivalent to magnetic control. Since DTT is a machine initially planned to test alternatives to ITER's detached condition exhaust, applicable to DEMO, it is natural that it could also be used as a platform to experiment and validate other DEMO solutions namely reflectometry. For DEMO, a series of reflectometry systems, poloidally distributed, will be used to measure the plasma position and shape. Reflectometry systems for density plasma evaluation presently installed probe the plasma from an equatorial position on the low field side or, on few cases, on the high field side (HFS), a configuration that is the most advantageous. All the accumulated experience of the reflectometry community is restricted to 
these setups. There is, therefore, a need to acquire expertise on profile reflectometry working out of the equatorial regions especially on the top and bottom positions of a tokamak where the difficulties will be more severe due to the plasma curvature and poloidal density gradients. A set of 5 reflectometers, a conventional one operating on the LFS and 4 on these more challenging positions, top LSF, bottom LFS, top HFS and bottom HFS, would provide an invaluable experimental setup to take reflectometry to the next level required by DEMO. Also, due to the early stage of diagnostic planning and implementation on DTT, the installation of such a system would be more feasible than on other existing machines. To support the installation of such systems, numerical simulation presents itself as a worthy tool and should be considered in the planning of these reflectometers. The choice of synthetic reflectometers made for this work, makes the proof of principle on the capability of the simulation tools to support the implementation of reflectometers at DTT, either it be the already planned conventional ones or alternative non-conventional configurations. The full-wave simulations performed in this work proved quite useful illustrating the possibility of reflectometry measurements not only in the conventional position $\left(\alpha=0^{\circ}\right)$ but especially on non-equatorial positions $\left(\alpha=-70^{\circ}\right)$.

\section{Acknowledgments}

This work has been carried out within the framework of the EUROfusion Consortium and has received funding from the Euratom research and training programme 2014-2018 and 2019-2020 under grant agreement No 633053 and within the framework of the French Federation for Magnetic Fusion Studies (FR-FCM). IST activities also received financial support from Fundação para a Ciência e Tecnologia through project UID/FIS/50010/2019. The views and opinions expressed herein do not necessarily reflect those of the European Commission.

\section{References}

[1] R. Albanese, DTT: a divertor tokamak test facility for the study of the power exhaust issues in view of DEMO, Nucl. Fusion 57 (2016) 016010.

[2] H.-J. Hartfuß, Fusion Plasma Diagnostics with mm-Waves, Chapter 4 Passive Diagnostics, John Wiley \& Sons (2013).

[3] S. Heuraux and F. da Silva, Simulations on wave propagation in fluctuating fusion plasmas for reflectometry applications and new developments, Discret. Contin. Dyn. S. S 5 (2011) 307.

[4] C. Lechte, Investigation of the scattering efficiency in doppler reflectometry by two-dimensional full-wave simulations, IEEE Trans. Plasma Sci. 37 (2009) 1099.

[5] L. Shi, E. J. Valeo, B. J. Tobias, G. J. Kramer, L. Hausammann, W. M. Tang et al., Synthetic diagnostics platform for fusion plasmas (invited), Rev. Sci. Instrum. 87 (2016) 11D303.

[6] F. da Silva, M. C. Pinto, B. Després and S. Heuraux, Stable explicit coupling of the yee scheme with a linear current model in fluctuating magnetized plasmas, J. Comput. Phys. 295 (2015) 24.

[7] F. da Silva, S. Heuraux, S. Hacquin and M. Manso, Unidirectional transparent signal injection in finite-difference time-domain electromagnetic codes-application to reflectometry simulations, J. Comput. Phys. 203 (2005) 467. 
[8] F. da Silva, S. Heuraux, B. Després and M. Campos Pinto, Numerical advances in kernels for FDTD Maxwell codes using the Yee algorithm, in proceedings of the $11^{\text {th }}$ International Reflectometry Workshop for fusion plasma diagnostics, Palaiseau, France, 22-24 April 2013.

[9] F. da Silva, S. Heuraux and T. Ribeiro, Introducing REFMULF, a $2 D$ full polarization code and REFMUL3, a 3D parallel full wave Maxwell code, in proceedings of the $13^{\text {th }}$ International Reflectometry Workshop for fusion plasma diagnostics, Daejeon, South Korea, 10-12 May 2017.

[10] F. da Silva, S. Heuraux, E. Ricardo, P. Quental and J. Ferreira, Assessment of the measurement performance of the in-vessel systems of gaps 4 and 6 of the ITER Plasma Position Reflectometer using a FDTD Maxwell full-wave code, in proceedings of the $13^{\text {th }}$ International Reflectometry Workshop for fusion plasma diagnostics, Daejeon, South Korea, 10-12 May 2017.

[11] R. Martone, R. Albanese, F. Crisanti, P. Martin and A. Pizzuto, DTT Divertor Tokamak Test faciliy Interim Design Report, ENEA Italian Agency for New Technologies, Energy and Sustainable Economic Development (2019).

[12] L. Frassinetti, M. Beurskens, S. Saarelma, J. Boom, E. Delabie, J. Flanagan et al., Global and pedestal confinement and pedestal structure in dimensionless collisionality scans of low-triangularity h-mode plasmas in JET-ILW, Nucl. Fusion 57 (2016) 016012. 